\title{
EFFECT OF DIFFERENT STORAGE MEDIA ON RE-ATTACHMENT OF INCISAL FRAGMENT: LABORATORY STUDY
}

\author{
Abeer M. Abdellatif*, Ibrahim H. El-Kalla* and Aya A. El-Gammal*
}

\begin{abstract}
Background: Tooth fragment re-attachment represents an important technique in restoring fractured anterior teeth. The esthetic appearance and the strength of the final restoration is dependent partly on correct hydration of the fragment.

Aim: Evaluate and compare the effect of different storage media of the tooth fragments on the color matching of the re-attached segment with the original tooth structure, on fracture resistance of re-attached teeth and on the mode of failure of re-attached fragments after one day, one week and two weeks storing time.

Design: A total number of 144 permanent incisor teeth were used. The fracturing procedure was done by a blunt chisel and hammer. The fractured fragments were then stored in appropriate storage media (dry, tap water, full humidity and milk) for one day, one week and two weeks. As scheduled, the fragments were re-attached using a flowable composite. After re-attachment of the fragments, standard photographs were captured for all teeth. Then, the photographs were assessed and the results were presented as percentages. All samples were subjected to fracture resistance test using a universal testing machine. The force required to fracture each tooth was recorded in Newton $(\mathrm{N})$ and the data were collected and statistically analysed. The fractured edges of the teeth and fragments were examined under a stereomicroscope and the results were tabulated.
\end{abstract}

Results: Fragments stored in dry environment demonstrated the highest color disharmony results even if the fragment kept for one day. One day fragment storing in milk and water was found to be with significantly higher fracture resistance than one and two weeks storing $(\mathrm{P}<0.001)$. No significant differences were detected between the different media in one and two weeks storing. The mode of failure revealed the adhesive mode of fracture at the re-attachment line in all groups.

Conclusion: Hydration and dryness avoidance are of fundamental importance for the maintenance of the original aesthetic appearance of the tooth. Keeping the tooth fragment wet in milk or water for one day yields the highest fracture resistance.

* Department of Pediatric Dentistry, Mansoura University, Mansoura, Egypt 


\section{INTRODUCTION}

Dental trauma is a very frequent accident among children and adolescents, usually associated with sport activities, falls and bicycle accidents. ${ }^{1}$ The treatment of coronal fracture is a considerable challenge for the dentist because it has to fulfil many parameters like a necessity to obtain an esthetic result comparable to the natural form. ${ }^{3}$ Despite the recent advances in adhesive materials and restorative technique, there is no restorative material that can reproduce the esthetic and functional needs as much as the natural dental structures. ${ }^{4}$ Therefore, came the concept of "tooth fragment re-attachment" (provided fragment available) making it possible for the dentist to use patient's own intact tooth fragment to restore the fractured tooth by re-attaching the fragment back to the tooth. ${ }^{5}$

In 1964 the concept of re-attachment was first described by Chosack and Eidelman ${ }^{6}$ Fragment reattachment procedure usually involves storage and preparation of the fragment prior to its re-attachment. These procedures are important determinants for the overall clinical outcome. ${ }^{7,8}$

The prognosis of the fragment re-attachment depends on the firm attachment of the fragment to the tooth remnant. Tooth preparation, impervious margins and strong bonding between the two segments are critical factors for firm re-attachment. ${ }^{3 \text {, }}$ ${ }^{9},{ }^{10}$ The strength of the final restoration is dependent also on correct hydration of the fragment so one of the factors that play an important role in the success of fragment re-attachment is the mode of the fragment storage following trauma. ${ }^{11-13}$ In many cases, immediate re-attachment of the fractured fragment is not allowed as the fractured tooth may need pulp treatment. As a limited number of studies have been performed on the hydrating media and the time of storing. So it is meaningful to test several preservative media to stand for the one that could aid in re-attaching the fragment perfectly.
The aim of this study is to evaluate and compare the effect of different storage media of the tooth fragments on the color matching of the re-attached segment with the original tooth structure, on fracture resistance of re-attached teeth and on the mode of failure of re-attached fragments after one day, one week and two weeks storing time.

\section{MATERIALS AND METHODS}

In this experimental laboratory study, a total number of 144 human permanent mandibular incisors freshly extracted for periodontal reason were used. The teeth were free from cracks, caries or any other kind of structural defects. The tissue remnants on the root surface of the teeth were removed with curettes and ultrasonic tips. The teeth were then stored in a plastic container with water and thymol $(0.2 \%$ thymol solution) till experimentation.

\section{Intentional coronal fracture}

A line was traced on the labial surface of each tooth, it should be $3 \mathrm{~mm}$ from the incisal edge and parallel to it. Then, each tooth was embedded in acrylic resin block up to three millimeters away from the marker so that the line axis of the tooth was parallel with the line axis of the acrylic block and the incisal edge was parallel with the horizontal line.

The fracturing procedures were done by using a blunt chisel (Single Bevel Straight Chisel $12 \mathrm{~mm}$ size) and hammer. The chisel was placed on the marked line perpendicularly to the labial surface of the tooth then a sudden stroke was directed to the head of the chisel with a hammer to fracture the incisor. The teeth displaying a fracture pattern different from the premeditated line or an unclear fracture line were discarded at this stage. Both the tooth remnant and incisal fragment of each tooth were then coded to ensure that each remnant could be matched correctly to its fragment at the time of fragment re-attachment. 


\section{Storage of fractured fragment}

Immediately after fracturing, the fractured fragments were stored in separate marked containers with appropriate storage medium (dry, milk, and saline) for $24 \mathrm{~h}$, one week and two weeks as scheduled and the remaining tooth structure along with acrylic blocks were stored again in distilled water until re-attachment, so the experimental groups were as follow:

Group A: Specimens stored dry in plastic containers: Group A1: Specimens stored dry in a plastic container for 24 hours. Group A2: Specimens stored dry in a plastic container for 1 week.Group A3: Specimens stored dry in a plastic container for 2 weeks. Group B: Specimens stored in $100 \%$ humidity in small closed plastic containers with a cotton soaked in tap water. Group B1: Specimens stored in $100 \%$ humidity for 24 hours. Group B2:Specimens be stored in 100\% humidity for 1 week.Group B3: Specimens stored in 100\% humidity for 2 weeks. Group C: Specimens stored in tap water. Group C1: Specimens stored in tap water for 24 hours. Group C2: Specimens in tap water for 1 week. Group C3: Specimens in tap water for 2 weeks. Group D: Specimens in milk (Full Cream Milk, Juhayna, EGYPT). Group D1: Specimens stored in milk for 24 hours. Group D2: Specimens stored in milk in a refrigerator for 1 week. Group D3: Specimens stored in milk in a refrigerator for 2 weeks.

\section{Re-attachment of the fragments}

Fragments were re-attached by means of simple re-attachment technique without any additional preparation of the fragment or tooth. The fractured parts were rinsed and dried to be ready for reattachment. The fractured surfaces of both the fragment and the tooth were etched by $37 \%$ phosphoric acid gel (Scotchbond Universal Etchant - Etching Gel, 3M ESPE, St. Paul, MN, USA) for 15 seconds and rinsed for 10 seconds followed by air drying for 5 seconds to remove excess water. Bonding agent (Adper Single Bond Plus Adhesive, 3M ESPE, St. Paul, MN, USA) was applied to the etched surfaces to be attached in two consecutive coats, and the surfaces were dried for 5 seconds with gentle air steam from an air syringe to allow solvent evaporation. The bonding agent was light cured for 20 seconds in the fractured fragment and 20 seconds in the tooth remnant $\left(600 \mathrm{~mW} \mathrm{~cm}^{-2}\right.$, Ivoclar vivadent LEDition, Austria). Flowable composite (Filtek flowable Z350, 3M ESPE, USA) was applied to the fractured surface of the fragment and tooth remnant then resin composite on each tooth surface (buccal and lingual) was light-cured for 40 seconds.

\section{Color matching}

After re-attachment of the fragments, a standard photograph was captured for each tooth using a fixed film-object distance and magnification. Each photograph was assessed according to the criteria; Score A: No noticeable difference from original teeth color Score B: Slight color mismatch. Score C: Obvious color mismatch. The results were presented as percentages.

\section{Fracture of restored teeth}

All the samples were then subjected to fracture strength test using a universal testing machine (Model LRX-plus; Lloyd Instruments Ltd., Fareham, UK) at a speed of $0.5 \mathrm{~mm} / \mathrm{min}$. The load was applied using a custom made load applicator (Mono-bevel chisel steel rod $0.6 \mathrm{~mm}$ thickness). The force application was always at $90^{\circ}$ with respect to the buccal surface and positioned exactly on the fractured line that was marked with a marker. The force required to fracture each tooth was recorded in Newtons (N). The data collected were tabulated accordingly and subjected to statistical analysis.

\section{Evaluation of mode of failure}

The fractured edges of the teeth and fragments were examined under a stereomicroscope (Olympus, 
New York, NY, USA) to determine the nature of failure that occurred during fracturing procedures in each group (cohesive, adhesive or mixed) and the results were tabulated.

\section{Statistical analysis}

Data were collected, tabulated and statistically analyzed with Statistical Package for Social Sciences (SPSS) version 21.13The normality of data was first tested with Shapiro-Wilk test. Continuous variables were presented as mean $\pm \mathrm{SD}$ (standard deviation). Analysis of Variance (ANOVA test) used for comparison of means of more than two groups. Repeated measure ANOVA tests used for comparison between three different durations. For color matching results kappa test was used for assessing reliability.

\section{RESULTS}

\section{Color matching}

Tables 1,2 and 3 show the color matching evaluation between the tooth structure and its incisal fragment for the twelve groups.

The results showed that Fragments stored in dry environment demonstrated the highest color disharmony results even if the fragment kept for one day. A total of $6(60 \%)$ fragments kept in milk for one day, $6(60 \%)$ fragments kept in humidity for one week and 7 (70\%) fragments kept hydrated in water for two weeks demonstrated no color disharmony.

\section{Fracture resistance of restored teeth}

Results were expressed as Mean \pm Standard Deviation (Table 4, 5, 6 and 7). ANOVA analysis was used for multiple group comparison. The $P$ value was calculated for statistical significance ( $\mathrm{P} \leq 0.05$ is statistically significant).

TABLE (1) Color matching among the groups according to the storage medium for 1 day storing time.

\begin{tabular}{|c|c|c|c|c|c|c|c|c|}
\hline \multirow{2}{*}{$\begin{array}{c}\text { Group 1 } \\
\text { 1 Day }\end{array}$} & \multicolumn{2}{|c|}{ A } & \multicolumn{2}{|c|}{ B } & \multicolumn{2}{c|}{ C } & \multicolumn{2}{c|}{ Total } \\
\cline { 2 - 9 } & No. & \% & No. & \% & No. & \% & No. & \% \\
1B: Dry & 1 & 10 & 1 & 10 & 8 & 80 & 10 & 100 \\
humidity & 3 & 30 & 4 & 40 & 3 & 30 & 10 & 100 \\
1C: Water & 2 & 20 & 6 & 60 & 2 & 20 & 10 & 100 \\
1D: Milk & 6 & 60 & 3 & 30 & 1 & 10 & 10 & 100 \\
\hline
\end{tabular}
A: No noticeable difference from original teeth color
B: Slight color mismatch
C : Obvious color mismatch

TABLE (2) Color matching among the groups according to the storage medium for 1 week storing time.

\begin{tabular}{|c|c|c|c|c|c|c|c|c|}
\hline \multirow{2}{*}{$\begin{array}{c}\text { Group 2 } \\
\text { 1Week }\end{array}$} & \multicolumn{2}{|c|}{ A } & \multicolumn{2}{|c|}{ B } & \multicolumn{3}{c|}{ C } & \multicolumn{2}{c|}{ Total } \\
\cline { 2 - 9 } & No. & $\%$ & No. & $\%$ & No. & $\%$ & No. & $\%$ \\
2A: Dry & 0 & 0 & 3 & 30 & 7 & 70 & 10 & 100 \\
2B: 100\% & 6 & 60 & 3 & 30 & 1 & 10 & 10 & 100 \\
humidity & & & & & & & & \\
2C: Water & 5 & 50 & 3 & 30 & 2 & 20 & 10 & 100 \\
2D: Milk & 3 & 30 & 5 & 50 & 2 & 20 & 10 & 100 \\
\hline
\end{tabular}


TABLE (3) Color matching among the groups according to the storage medium for 2 weeks storing time.

\begin{tabular}{|l|c|c|c|c|c|c|c|c|}
\hline \multicolumn{1}{|c|}{$\begin{array}{l}\text { Group 3 } \\
\text { 2Weeks }\end{array}$} & \multicolumn{2}{|c|}{ A } & \multicolumn{2}{c|}{ B } & \multicolumn{3}{c|}{ C } & \multicolumn{2}{c|}{ Total } \\
\cline { 2 - 9 } & No. & \% & No. & \% & No. & $\%$ & No. & \% \\
\hline 3A: Dry & 0 & 00 & 2 & 20 & 8 & 80 & 10 & 100 \\
3B: 100\% & 3 & 30 & 4 & 40 & 3 & 30 & 10 & 100 \\
humidity & & & & & & & & \\
3C: Water & 7 & 70 & 2 & 20 & 1 & 10 & 10 & 100 \\
3D: Milk & 4 & 40 & 3 & 30 & 3 & 30 & 10 & 100 \\
\hline
\end{tabular}

TABLE (4) Mean fracture resistance for each group according to the storage medium for 1 day fragment storing.

\begin{tabular}{|c|c|c|c|c|}
\hline \multirow[t]{2}{*}{ Environment } & \multicolumn{4}{|c|}{ Fracture force } \\
\hline & Mean $\quad \pm$ & SD & Min & Max \\
\hline Dry & $229.58^{\mathrm{A}}$ & 35.36 & 153.13 & 272.12 \\
\hline $100 \%$ Humid & $243.88^{\mathrm{A}}$ & 49.77 & 159.19 & 318.60 \\
\hline Water & $276.68^{\text {в }}$ & 35.11 & 235.61 & 342.58 \\
\hline Milk & $331.12^{\mathrm{C}}$ & 19.37 & 300.25 & 364.59 \\
\hline $\begin{array}{l}\text { Test of sig. } \\
\text { p-value }\end{array}$ & \multicolumn{4}{|c|}{$\begin{array}{c}\mathrm{F}=18.276 \\
\mathrm{P}=\leq .001\end{array}$} \\
\hline
\end{tabular}

* Groups with different superscript letters are statistically significantly different according to the Tukey HSD test ( $p \leq 0.05)$.

TABLE (5) Mean fracture resistance of each group according to the storage medium for 1 week fragment storing.

\begin{tabular}{|l|c|c|c|c|}
\hline \multirow{2}{*}{ Environment } & \multicolumn{4}{|c|}{ Fracture force } \\
\cline { 2 - 5 } & Mean \pm & SD & Min & Max \\
\hline Dry & 186.34 & 54.95 & 109.29 & 299.93 \\
\hline $100 \%$ Humid & 219.85 & 52.97 & 132.43 & 293.34 \\
\hline Water & 221.84 & 60.75 & 158.59 & 348.21 \\
\hline Milk & 225.74 & 28.52 & 192.24 & 283.88 \\
\hline $\begin{array}{l}\text { Test of sig. } \\
\text { p-value }\end{array}$ & \multicolumn{3}{|c|}{$\begin{array}{c}\mathrm{F}=1.544 \\
\mathrm{P}=.216\end{array}$} \\
\hline
\end{tabular}


TABLE (6) Mean fracture resistance for each group according to the storage medium for 2 weeks storing.

\begin{tabular}{|c|c|c|c|c|}
\hline \multirow{2}{*}{ Environment } & \multicolumn{4}{|c|}{ Fracture force } \\
\cline { 2 - 5 } & Mean \pm & SD & Min & Max \\
\hline Dry & 194.62 & 51.90 & 120.56 & 295.19 \\
\hline $100 \%$ Humid & 204.47 & 42.76 & 152.19 & 344.34 \\
\hline Water & 215.47 & 68.09 & 156.38 & 360.45 \\
\hline Milk & 230.79 & 60.77 & $\begin{array}{c}\mathrm{F}=.899 \\
\mathrm{P}=.449\end{array}$ \\
\hline $\begin{array}{c}\text { Test of sig. } \\
\text { p-value }\end{array}$ & \multicolumn{2}{|}{} \\
\hline
\end{tabular}

TABLE (7) Comparison between mean fracture resistance among the groups kept in the same medium at different times.

\begin{tabular}{|c|c|c|c|c|c|c|c|}
\hline \multirow{2}{*}{ Environment } & \multicolumn{2}{|c|}{ Day 1} & \multicolumn{2}{|c|}{ Week1 } & \multicolumn{2}{|c|}{ Week2 } & \multirow{2}{*}{$\begin{array}{c}\text { Test of sig. } \\
\text { p-value }\end{array}$} \\
\hline & Mean \pm & SD & Mean \pm & SD & Mean \pm & SD & \\
\hline Dry & $229.58^{\mathrm{A}}$ & 35.36 & $186.34^{\mathrm{B}}$ & 54.95 & 194.62 & 51.90 & $\begin{array}{c}\mathrm{F}=2.723 \\
\mathrm{P}=.080\end{array}$ \\
\hline $100 \%$ Humid & $243.88^{\mathrm{A}}$ & 49.77 & 219.85 & 52.97 & $204.47^{\text {в }}$ & 42.76 & $\begin{array}{c}\mathrm{F}=1.998 \\
\mathrm{P}=.152\end{array}$ \\
\hline Water & $276.68^{A}$ & 35.11 & $221.84^{\text {в }}$ & 60.75 & $215.47^{\mathrm{CB}}$ & 68.09 & $\begin{array}{c}\mathrm{F}=4.263 \\
\mathrm{P}=.023\end{array}$ \\
\hline Milk & $331.12^{\mathrm{A}}$ & 19.37 & $225.74^{\text {в }}$ & 28.52 & $230.79^{\mathrm{CB}}$ & 60.77 & $\begin{array}{c}\mathrm{F}=26.049 \\
\mathrm{P}=.000\end{array}$ \\
\hline
\end{tabular}

* Groups with different superscript letters are statistically significantly different according to the Tukey HSD test (p $\leq 0.05)$.

The results showed that the milk group demonstrated the highest fracture resistance followed by the water group then the $100 \%$ humidity group and the dry group which had the least fracture resistance. One day fragment storing in milk and water was found to be with significantly higher fracture resistance than one and two weeks storing $(\mathrm{P}<0.001)$. No significant differences were detected between the different media in one and two weeks storing.

\section{Evaluation of mode of failure}

The results of the stereomicroscopic examination for the mode of failure at the fracture site of teeth in different groups revealed the adhesive mode of fracture at the re-attachment line in all groups.

\section{DISCUSSION}

Although tooth fractures are much more common in maxillary incisors, human mandibular incisors were used in this study, because they were obtained in sufficiently large numbers from patients due to periodontal reasons and have little difference and variation in their dimensions. ${ }^{14,15}$ In this study, thymol ( $0.2 \%$ thymol solution) was used as storage medium for the collected teeth because it has been widely assumed that water with thymol have no effect on either the organic or inorganic content of dentin. X-ray diffraction has failed to show changes in the mineral content of dentin after thymol storage. ${ }^{16}$

The teeth were fractured by a blunt chisel and hummer to simulate the fracture pattern, surface 
topography and the micromechanical adaptation of the surfaces under clinical conditions. ${ }^{17}$ Badami et al. ${ }^{18}$ and Reis et al. ${ }^{9}$ have reported that the surface of a sectioned tooth is different from a naturally occurring fractured one, as the fracture pattern produces fragments with a good fitting.

The selection of the storage conditions in this study (dry, tap water, full humidity and milk) was based on publicly accessible materials. Milk was selected as when the tooth is avulsed, parents are advised to store it in milk. Therefore, in case of other tooth injuries such as a crown fracture, some parents tend to keep the fragment in milk. ${ }^{15}$ The selected storage durations (one day, one week and two weeks) were intended to resemble different possibilities in actual practice as in many cases the fractured tooth may need pulp treatment before fragment re-attachment. On other hand, it considered to simulate various times from the incident until the patient may consult a dentist.

In this study major changes were seen in the appearance of the teeth after re-attachment that its fragments were stored in the dry environment. That is in agreement with Toshihiro et al. ${ }^{13}$ study who observed that the crown fragment was much more white than the fractured tooth because of dehydration of the underlying dentin as the fragment was kept dry for 12 days before re-attachment and it was so difficult to mask the color disharmony between the fractured tooth and the re-attached fragment with composite resin. These results also correspond with those of Yilmaz et al. ${ }^{19}$ who observed initial color disharmony in restored teeth after fragment re-attachment when the fragment is kept in dry environment prior to its re-attachment.

Among the tested groups, it was noted that fragments which were kept in the dry environment before re-attachment had the least mean fracture strength values. These results are consistent with a study carried out by Capp et al. ${ }^{20}$ who also showed that the strength of the hydrated and rehydrated bonded fragments was greater than that of the dehydrated fragments. Keeping the fragments in a moist environment ensured that there is no or minimal collapse of the collagen fibers in the dentin leading to a better bond strength. These results also support Shirani et al. ${ }^{15}$ finding who reported that loss of dentin moisture and its shrinkage results in reduction of the composite surface contact with dentin. Besides, over acid-etching may occur and result in unfavorable effects on the bonding condition.

In the experimental groups in which their fragments were kept in $100 \%$ humidity or water, their bond strength increased more than those of the groups whose fractured parts were kept in a dry environment. It seems that in these two environments, little osmotic and dimensional changes happen in the dentin surface and stronger bond strength is achieved. This ensures the important role of moisture in the bonding mechanism.

Fragments stored in milk for one day gave the highest mean fracture strength values. The best storage environment, as observed in this study, is milk. It has been proved that milk elements such as calcium and phosphate can harden and stiffen both demineralized and healthy dentin by permeating the surface. ${ }^{21}$ This is probably the reason why enhancement of bond strength was observed in milk group which was rich in calcium and phosphates..$^{15}$ These results are in agreement with those of Shirani et al. ${ }^{15}$ who found the best results is obtained when the fragment is kept in milk or saliva.

For the fragments which were kept in different media for one day there was a highly statistically significant difference in results between different groups, but for those which were kept for one or two weeks the results revealed that there was no statistically significant difference between groups. This in agreement with Sharmin \& Tomas $^{22}$ findings who reported that if the storage time was more than 48 hours, even the samples stored in milk could 
have obtained similar fracture resistance as stored in saline. It can be due to the fragments reach full hydration with time.

No significant difference between fracture resistances of teeth groups kept dry for different times is seen in the present study. These results correspond with those of Shirani et al. ${ }^{17}$ who also showed that the dehydration process appears to have approached a plateau-like trend within six hours and any further drying (up to three days) seems to be almost ineffective in the final strength of the tooth.

Comparing the mode of failure, all samples in all groups showed adhesive mode of fracture (failure at tooth surface and restoration interface). All the specimens failed at the weakest point, which in this case proved to be the re-attachment line. Since the specimens can be considered as consisting of three components, the restoration cracked at the tooth material interface could be explained by the fact that the first layer (tooth) was immobilized in the holder of the testing instrument and the other two layers (the restorative material and the fragment) were free. Another possible explanation is the lack of a perfect fitting, a discrepancy between the dental fragments may represent an area of increased stress. $^{22,23}$

\section{REFERENCES}

1. Flores MT, Andreasen JO, Bakland LK, Feiglin B, Gutmann JL, Oikarinen K, Ford TR, Sigurdsson A, Trope M, Vann WF Jr. International Association of Dental Traumatology Guidelines for the evaluation and management of traumatic dental injuries. Dent Traumatol. 2001; 5:193-198.

2. Castro JC, Poi WR, Manfrin TM, Zina LG. Analysis of the fractures and crown-root fractures due to dental trauma assisted by the Integrate Clinic from 1992 to 2002. Dent Traumatol. 2005; 21:121-126.

3. Demarco FF, Fay RM, Pinzon LM, Powers JM. Fracture resistance of reattached coronal fragments-influence of different adhesive materials and bevel preparation. Dent Traumatol. 2004; 20:157-63.

4. Alvares I, Sensi LG, Araujo EM Jr, Araujo E. "Silicone index: an alternative approach for tooth fragment reattachment," Journal of Esthetic and Restorative Dentistry. 2007; 19:240-245.

5. Hedge RJ. Tooth fragment reattachment - an esthetic alternative: report of a case. Incisal edge reattachment: indication for use and clinical technique. Br Dent J.1999; 186:614-619.

6. Cited from: Ajay Choudhary, Rakesh Garg, Anindya Bhalla, Rohit Kumar Khatri. Tooth fragment reattachment: An esthetic, biological restoration. Nat Sci Biol Med J. 2015; 6:205-207.

7. Andreasen FM and Andreasen JO. Crown fractures. In: Textbook and color atlas of traumatic injuries to the teeth, 4th edn. Oxford, UK: Blackwell Publishing Ltd. 2007; 280-313.

8. Farik B, Munksgaard EC, Andreasen JO, Kreiborg S. Drying and rewetting anterior crown fragments prior to bonding. Endod Dent Traumatol. 1999; 15:113-116.

9. Reis A, Francci C, Longuerco AD, Carrilho MR, Rodrigues Filho LE. Re- Attachment of anterior fractured teeth: fracture strength using different techniques. Oper Dent. 2001; 26:287-294.

10. Prabhakar AR, Kurthukoti A, Kayalvizhi G. A comparison of impact strength of fragment-bonded anterior teeth using 3 different restorative materials: an in-vitro study. J Indian Soc Pedod Prev Dent. 2007; 25:88-92.

11. Simonsen RJ. Restoration of a fractured central incisor using original tooth fragment. J Am Dent Assoc. 1982; 105:646-648.

12. Baratieri LN, Monteiro Júnior S, Caldeira de Andrada MA. The "sandwich" technique as a base for reattachment of dental fragments. Quintessence Int. 1991; 22:81-85.

13. Toshihiro K, Rintaro T. Rehydration of crown fragment 1 year after reattachment: a case report. Dent Traumatol. 2005; 21:297-300.

14. Sengun A, Ozer F, Unlu N, Ozturk B. Shear bond strengths of tooth fragments reattached or restored. J Oral Rehabil. $2003 ; 30: 82-86$.

15. Shirani F, Malekipour MR, Tahririan D, manesh VS. Effect of storage environment on the bond strength of reattachment of crown fragments to fractured teeth. J Conserv Dent. 2011; 14:269-272.

16. Goodis HE, Marshall GW Jr, White JM. The effects of storage after extraction of the teeth on human dentine permeability in vitro. Arch Oral Biol. 1991; 36:561-566. 
17. Shirani F, Malekipour MR, Manesh VS, Aghaei F. Hydration and dehydration periods of crown fragments prior to reattachment. Oper Dent. 2012; 37:501-508.

18. Badami AA, Dunne SM, Scheer B. An in vitro investigation into the shear bond strengths of two dentine bonding agents used in the reattachment of incisal edge fragments. Endod Dent Traumatol. 1995; 11:129-135.

19. Yilmaz Y, Guler C, Sahin H, Eyuboglu O. Evaluation of tooth-fragment reattachment: a clinical and laboratory study. Dental Traumatology. 2010; 26:308-314.

20. Capp CI, Roda MI, Tamaki R, Castanho GM, Camarg MA, Alberto de Cara AA. Reattachment of rehydrated dental fragment using two techniques. Dental Traumatology. 2009; 25:95-99.

21. O’Donnell JN, Schumacher GE, Antonucci JM, Skrtic D. Adhesion of amorphous calcium phosphate composites bonded to dentin: A study in failure modality. J Biomed Mater Res B Appl Biomater. 2009; 90:238-2349.

22. Sharmin DD, Thomas E. Evaluation of the effect of storage medium on fragment reattachment. Dent Traumatol. 2012; 29(2):99-102.

23. Kovacs M, Pacurar M, Pop M, Blanka P, Bukhari C. Fracture resistance of tooth fragments reattached with different techniques. an in vitro study. Romanian Journal of Oral Rehabilitation. 2012; 4:36-41. 